\title{
Effects of thiourea on the skull of Triturus newts during ontogeny
}

\author{
Maja Ajduković ${ }^{\text {Corresp., } 1}$, Tijana Vučić ${ }^{2}$, Milena Cvijanović ${ }^{1}$ \\ ${ }^{1}$ Department of Evolutionary Biology, Institute for Biological Research "Siniša Stanković", National Institute of the Republic of Serbia, University of \\ Belgrade, Belgrade, Serbia \\ 2 Institute of Zoology, Faculty of Biology, University of Belgrade, Belgrade, Serbia \\ Corresponding Author: Maja Ajduković \\ Email address: maja.ajdukovic@ibiss.bg.ac.rs
}

\section{Background}

In amphibians, thyroid hormone (TH) has a profound role in cranial development, especially in ossification of the late-appearing bones and remodeling of the skull. In the present study, we explored the influence of TH deficiency on bone ossification and resulting skull shape during the ontogeny of Triturus newt hybrid larvae obtained from interspecific crosses between $T$. ivanbureschi and $T$. macedonicus.

\section{Methods}

Larvae were treated with two concentrations of thiourea (an endocrine disruptor that chemically inhibits synthesis of TH) during the midlarval and late larval periods. Morphological differences of the cranium were assessed at the end of the midlarval period (ontogenetic stage 62) and the metamorphic stage after treatment during the late larval period.

\section{Results}

There was no difference in the ossification level and shape of the skull between the experimental groups (control and two treatment concentrations) at stage 62. During the late larval period and metamorphosis, TH deficit had a significant impact on the level of bone ossification and skull shape with no differences between the two treatment concentrations of thiourea. The most pronounced differences in bone development were: the palatopterygoid failed to disintegrate into the palatal and pterygoid portions, retardation was observed in development of the maxilla, nasal and prefrontal bones and larval organization of the vomer was retained in thiourea-treated larvae.

\section{Conclusions}

This implies that deficiency of TH caused retardation in development and arrested metamorphic cranium skeletal reorganization, which resulted in divergent cranial shape compared to the control group. Our results confirmed that skull remodeling and ossification of late-appearing bones is $\mathrm{TH}$-dependent, as in other studied Urodela species. Also, our results indicate that TH plays an important role in the establishment of skull shape during the ontogeny of Triturus newts, especially during the late larval period and metamorphosis, when TH concentrations reach their maximum. 
1

2

3

4

5

6

7 'Department of Evolutionary Biology, Institute for Biological Research "Siniša Stanković",

8 National Institute of the Republic of Serbia, University of Belgrade, Belgrade, Serbia

9 Institute of Zoology, Faculty of Biology, University of Belgrade, Belgrade, Serbia

Corresponding Author:

Maja Ajduković ${ }^{1}$

Bulevar despota Stefana 142, Belgrade, 11000, Serbia

Email address: maja.ajdukovic@ibiss.bg.ac.rs 
16

17

18

19

20

21

22

\section{Abstract}

\section{Background}

In amphibians, thyroid hormone (TH) has a profound role in cranial development, especially in ossification of the late-appearing bones and remodeling of the skull. In the present study, we explored the influence of TH deficiency on bone ossification and resulting skull shape during the ontogeny of Triturus newt hybrid larvae obtained from interspecific crosses between $T$. ivanbureschi and T. macedonicus.

\section{Methods}

Larvae were treated with two concentrations of thiourea (an endocrine disruptor that chemically inhibits synthesis of TH) during the midlarval and late larval periods. Morphological differences of the cranium were assessed at the end of the midlarval period (ontogenetic stage 62) and the metamorphic stage after treatment during the late larval period.

\section{Results}

There was no difference in the ossification level and shape of the skull between the experimental groups (control and two treatment concentrations) at stage 62. During the late larval period and metamorphosis, TH deficit had a significant impact on the level of bone ossification and skull shape with no differences between the two treatment concentrations of thiourea. The most pronounced differences in bone development were: the palatopterygoid failed to disintegrate into the palatal and pterygoid portions, retardation was observed in development of the maxilla, nasal and prefrontal bones and larval organization of the vomer was retained in thiourea-treated larvae.

\section{Conclusions}

This implies that deficiency of TH caused retardation in development and arrested metamorphic cranium skeletal reorganization, which resulted in divergent cranial shape compared to the 
41 control group. Our results confirmed that skull remodeling and ossification of late-appearing

42 bones is TH-dependent, as in other studied Urodela species. Also, our results indicate that $\mathrm{TH}$

43 plays an important role in the establishment of skull shape during the ontogeny of Triturus

44 newts, especially during the late larval period and metamorphosis, when TH concentrations reach

45 their maximum.

46 


\section{Introduction}

48

49

Thyroid hormones (thyroxine T4 and triiodothyronine T3), produced by the thyroid gland, play an important and widespread role in skeletal differentiation and morphogenesis in vertebrates (Hanken \& Summers, 1988; Rose \& Reiss, 1993; Smirnov \& Vassilieva, 2003a). In amphibians, thyroid hormone (TH) has a crucial role in metamorphosis, which represents dramatic structural and functional changes of larval tissue to the juvenile form (Kollros, 1961; Dodd \& Dodd, 1976). It is well known that TH synthesis and release to the bloodstream increases towards metamorphosis, reaches a maximum at the climax stage and decreases after metamorphosis (Regard, Taurog \& Nakashima, 1978; Larras-Regard, Taurog \& Dorris, 1981; White \& Nicoll, 1981; Alberch, Gale \& Larsen, 1986; Norman, Carr \& Norris, 1987; Sternberg et al., 2011). Also, TH plays a profound role in the skeletal development of amphibians, especially in remodeling of the skull, which undergoes abrupt changes during metamorphosis (Rose, 2003; Smirnov \& Vassilieva, 2003a). During amphibian ontogeny, the timing of cranial bone ossification shows species-specific characteristics and is TH-mediated (Rose \& Reiss, 1993; Smirnov \& Vassilieva, 2003b). Ossification sequences of cranial bones are therefore determined by TH concentration and by the sensitivity of osteogenic sites to TH (Smirnov \& Vassilieva, 2003b). According to Smirnov \& Vassilieva (2003a), bones appearing early in the skull (coronoid, palatine, dentary, vomer, premaxilla, squamosal, angular, pterygoid, parasphenoid), ones appearing later (parietal and frontal), and midlarval bones (exoccipital, orbitosphenoid, quadrate and prootic) are not TH-inducible. However, late-appearing bones such as the maxilla, prefrontal and nasal, as well as metamorphic skull remodeling, are TH-inducible (Smirnov \& Vassilieva, 2003a; Smirnov, Vassilieva \& Merkulova, 2011). 
only with exogenous $\mathrm{TH}$ but also with the goitrogenic substance thiourea, a chemical inhibitor of thyroid synthesis (Smirnov \& Vassilieva, 2003a, 2003b; Smirnov, Vassilieva \& Merkulova, 2011). Thiourea (CAS Number: 62-56-6) is well established in treating hyperactivity of the thyroid gland (hyperthyroidism) and causes a decrease in the level of TH (Gobdon, Goldsmith \& Charipper, 1943; Wheeler, 1953; Chakraborty et al., 2018). This anti-thyroid agent inhibits TH synthesis by the thyroid gland but does not block it completely, like the action of thyroidectomy, which represents surgical removal of the thyroid gland (Brown, 1997). The mechanism of antithyroid agents is realized via action on inhibition of thyroid peroxidase (TPO) (Engler, Taurog \& Nakashima, 1982; Wegner, Browne \& Dix, 2016), the enzyme responsible for oxidation of iodide in the biosynthesis of thyroid hormones. In the presence of iodine, thiourea competes with tyrosine residues of thyroglobulin (the precursor of thyroid hormones) for oxidized iodine, thus diverting oxidized iodine away from thyroglobulin (Sheikh \& McGregor, 1997). At last, antithyroid drugs themselves are oxidized and degraded. The use of thiourea, a well-known endocrine disruptor, revealed a strong inhibitory effect on thyroid hormone synthesis in amphibians (Takamura et al., 2020). Prolonged treatment with thiourea results in a hypertrophic and hyperplastic thyroid gland due to the pituitary gland's overproducing thyroid-stimulating hormone (TSH) in response to the drop in the level of TH in the blood. An extensive literature is available for all tetrapod groups concerning the effects of thiourea on morphology and physiology of the thyroid gland. Among amphibians, the majority of studies treated the ability of thiourea to inhibit metamorphosis in tadpoles (Gobdon, Goldsmith \& Charipper, 1943; Lynn, 1948; Steinmetz, 1950; Krishnapriya et al., 2014) and its influence on cranial osteology of larvae and newly metamorphosed newts under conditions of an artificially caused deficit of TH 
93 (Smirnov \& Vassilieva, 2003a, 2003b; Smirnov \& Vassilieva, 2005; Smirnov, Vassilieva \&

94 Merkulova, 2011). Despite numerous studies dealing with the influence of TH on induction of

95 the appearance of cranial bones and ossification in Urodela (Rose, 1995a, 1995b; Smirnov \&

96 Vassilieva, 2003a, 2003b; Smirnov, Vassilieva \& Merkulova, 2011), there have been no direct

97 studies on the mediation of skull shape by TH in Urodela.

98 The order Urodela is one of the major extant orders of the class Amphibia. It includes

99 salamanders and newts which, are characterized by slender bodies, blunt snouts, short limbs and

100 the presence of a tail in both larvae and adults. The genus Triturus forms a well-supported

101 monophyletic clade of newts within the family Salamandridae (Steinfartz et al., 2007; Wielstra \&

102 Arntzen, 2011). According to current taxonomy, the genus Triturus consists of nine species: two

103 marbled (T. marmoratus and T. pygmaeus) and seven crested (T. karelinii, T. ivanbureschi, T.

104 anatolicus, T. macedonicus, T. carnifex, T. cristatus, and T. dobrogicus) (Wielstra et al., 2019)

105 newt species.

106 The present study aimed to investigate whether altered levels of TH during larval

107 ontogeny have an impact on cranial morphology in Triturus newt hybrid larvae. We set up two

108 experiments. In the first experiment, larvae were treated with a goitrogenic substance during the

109 midlarval period and examined at the end of this period, at the ontogenetic stage 62 . In the

110 second experiment, larvae were treated during the late larval period and metamorphosis and

111 examined at the metamorphic stage. At these stages, we obtained the level of bone ossification,

112 estimated skull shape and compared them in two thiourea-treated groups and control. We

113 expected that a TH deficit would have a major impact on cranial morphology during the late

114 larval period and metamorphosis, when thyroid hormones reach their maximum level and have a

115 crucial role in the development of late-appearing bones. Accordingly, we expected that 
116 differences in cranial shape between treated and control groups will be pronounced at the

117 metamorphic stage.

118

119

\section{Materials \& methods}

120

121

122

123

124

125

126

127

128

129

130

131

132

133

134

135

136

137

138

139

\section{Animal housing}

We used hybrids obtained from crosses between T. ivanbureschi and T. macedonicus. These two species hybridize at their contact zones in a natural population (Arntzen, Wielstra \& Wallis, 2014; Wielstra et al., 2017). In natural unimodal hybrid populations, hybrids cannot be distinguished based on external morphology from parental species (Arntzen et al., 2018).

Additionally, it has been shown in the laboratory that values of tail shape, size and colouration patterns (Vučić et al., 2018), as well as general head shape during larval development and after metamorphosis (Vučić et al., 2019), are intermediate between hybrids and the parental species during ontogeny (Vučić et al., 2019). Taking into account the aforementioned, we decided to use hybrid larvae (obtained from experimental crosses) to explore possible differences in cranial morphology due to different $\mathrm{TH}$ levels during larval development, since we were not interested in interspecific divergence at this point.

After mating in common containers, gravid females were transferred separately to aquaria containing plastic strips for egg deposition. Eggs were collected daily and kept submerged in dechlorinated tap water in plastic Petri dishes until hatching. After that, hatchlings were transferred to a small plastic cup with dechlorinated tap water, where they developed in laboratory conditions under a natural day-night light regime at air temperatures of $18^{\circ} \pm 1^{\circ} \mathrm{C}$ and a water temperature of $16^{\circ} \pm 1^{\circ} \mathrm{C}$. Larvae were fed ad libitum with Artemia sp. at earlier developmental stages and with Tubifex sp. at later stages. The stage of larvae was determined

Peer] reviewing PDF | (2020:11:55888:2:1:REVIEW 1 May 2021) 
140 according to the developmental staging table for crested newts (Glücksohn, 1931), which is

141 based on limb development and digit formation (described only by external features). A degree

142 of limb differentiation appears to be suitable for ontogenetic staging under different TH regimes

143 because limb development is TH independent. For larval developmental stage 50, the basic

144 features are formation of the fourth digit in forelimbs and initiation of hindlimb bud formation.

145 Developmental stage 62 is characterized by fully developed limbs, formed external gills and the 146 appearance of larval skin pigmentation, unlike metamorphic individuals, which are characterized

147 by the resorption of external gills and closure of gill slits. Also, dramatic changes are notable in 148 the skin, especially in its pigmentation and thickness, in metamorphic individuals. A brief overview of developmental stages from hatching until metamorphosis with graphic presentation

150 of the analyzed stages is given in Table S1.

151

152

153

154

155

156

157

158

159

160

161

162

163

Experimental settings

A detailed protocol for the experimental design and an analysis plan suiting the research questions posed were prepared in advance. The first experiment started at larval stage 50 and lasted throughout the middle larval period until the larvae reached stage 62 (Glücksohn, 1931; see Table $\mathrm{S} 1)$. When the larvae $(\mathrm{N}=26)$ reached stage 50 , they were transferred to 2-L plastic containers half-filled with different media depending on the experimental group. Three groups were defined: 1) control (dechlorinated tap water), 2) low thiourea concentration $(0.05 \%$ solution of thiourea) and 3$)$ high thiourea concentration ( $0.1 \%$ solution of thiourea). Thiourea (p.a. $\geq 99.0 \%$; Sigma, St. Louis, MO, USA) solutions (low and high concentrations) were prepared in dechlorinated tap water. These two concentrations of thiourea were established in previous work on salamanders (Wheeler, 1953). The duration of exposure to thiourea was approximately three months for each experiment. To minimize confounding effects, the larvae of 
164 the control and two treatment groups were kept under the same laboratory conditions at the same

165 density (five larvae per container). The medium was changed every second day. When larvae

166 reached stage 62 (Glücksohn, 1931; Table S1), they were euthanized with ethyl 3-aminobenzoate

167 methanesulfonate better known as MS 222 (CAS Number: 886-86-2; Sigma, St. Louis, MO,

168 USA), and preserved whole in $96 \%$ ethanol.

169 The second experiment started at stage 62 and lasted throughout the late larval period 170 until larvae $(\mathrm{N}=49)$ from the control group complete metamorphosis. Because thiourea inhibits

171 TH synthesis and therefore inhibits the ability of larvae to metamorphose, sampling of larvae

172 was based on normally metamorphosing individuals in the control group. Metamorphosed

173 individuals are characterized by the resorption of external gills and closure of gill slits. Also,

174 some differences in the skin are notable, especially in pigmentation and thickness (Glücksohn,

175 1931; Table S1). Photos of cleared and stained skulls of analyzed larvae at the beginning and end

176 of the second experiment are given as Supplemental Fig. 1. The same experimental design, viz.,

177 control group and two concentrations of thiourea [low $(0.05 \%)$ and high $(0.1 \%)$, was used for

178 the second experiment. For the second experiment, there were three larvae per container to avoid

179 potential high larval density caused by the increase in larval body mass and size, which

180 invariably leads to some detrimental effects, such as reduction in larval growth or size at

181 metamorphosis (Altwegg, 2003). Larvae were reared in 2-L plastic containers half-filled with

182 thiourea media, while the control group was raised in dechlorinated tap water until the end of

183 metamorphosis, when all animals were sampled, euthanized and preserved whole in $96 \%$

184 ethanol. There were no differences in dissolved oxygen, $\mathrm{pH}$ and temperature between the control

185 group and two thiourea concentrations $(0.05$ and $0.1 \%)$. Also, the mortality rate and other growth

186 effects were similar in the control group and at two thiourea concentrations. All animals used in 
187 both experiments were included in the analysis and randomly allocated to treatment and control 188 groups.

All analysed larvae were prepared as skeletal whole-mounts. Materials were cleared with

190

191

192

193

194

195

196

197

198

199

200

201

202

203

204

205

206

207

208

209

trypsin and $1 \% \mathrm{KOH}$ and double-stained for cartilage and bone with Alcian blue and alizarin red S, respectively (Dingerkus \& Uhler, 1977). Whole-mounts of larvae were stored in the batrachological collection of the "Siniša Stanković" Institute for Biological Research. Collection of adult animals from natural populations was approved by the Ministry of Energy, Development and Environmental Protection of the Republic of Serbia (permit no. 35301-75/2014-08) and the Environmental Protection Agency of Montenegro (permit no. UPI328/4). The experiment was approved by the Ethical Committee of the "Siniša Stanković" Institute for Biological Research (decision no. 02-07/19). All experimental animals were treated in compliance with a European directive (2010/63/EU) on the protection of animals used for experimental and other scientific purposes.

Data collection

We used the minimal sample size per group (an individual larva was treated as an experimental unit) in order to be able to perform a valid statistical analysis. The cleared and stained skulls of analyzed larvae for stage $62(\mathrm{~N}=26)$ and the metamorphic stage $(\mathrm{N}=49)$ were photographed against a scale bar $(10 \mathrm{~mm})$ in Petri dishes positioned in the centre of the optical field using a Nikon Digital Sight Fi2 camera attached to a Nikon SMZ800 stereo zoom microscope. Skull photographs of high resolution were used for scoring the level of cranial element ossification and skull shape.

We scored the presence and ossification level of skeletal elements in the skull at two developmental stages for both the dorsal and the ventral side. Scores were in the range of 0 to 2 , 
210 where 0 represents the absence of bone, 1 stands for partially ossified bones and 2 represents

211 fully ossified bones. At stage 62, ossification scores were obtained from 10 larvae not treated

212 (control), 10 larvae treated with $0.05 \%$ thiourea (low concentration) and six larvae treated with

$2130.1 \%$ thiourea (high concentration). For that developmental stage, we scored the exoccipital,

214 frontal, parietal, premaxilla and squamosal bones on the dorsal skull side, and the

215 palatopterygoid, parasphenoid and vomer bones on the ventral side of the skull.

216 At the metamorphic stage, ossification scores were obtained from 14 individuals in the

217 control group, 19 individuals treated with $0.05 \%$ thiourea (low concentration) and 16 individuals

218 treated with $0.1 \%$ thiourea (high concentration). For that developmental stage, we scored the

219 frontal, maxilla, nasal, parietal, prefrontal and squamosal bones on the dorsal skull side, and the

220 palatopterygoid, pterygoid and vomer bones on the ventral side of the skull.

221

At stage 62, a configuration of eight two-dimensional landmarks for both the dorsal and

222 the ventral cranium was used to describe larval skull shape. For the metamorphic stage, 10 two-

223 dimensional landmarks for the dorsal and eight two-dimensional landmarks for the ventral

224 cranium were used (Fig. 1). A brief anatomical description of the position of cranium landmarks

225 for both stages is given in Table S2. All landmarks were digitized by the same person (MA)

226 using TpsDig software (Rohlf, 2005). Centroid size (CS) as a measure of size was calculated in

227 the CordGen program from the IMP package (Sheets, 2000). Generalized Procrustes analysis

228 (GPA) was employed to obtain shape coordinates (Procrustes coordinates) (Rohlf \& Slice, 1990;

229 Dryden \& Mardia, 1998) using the Morpho J software package (Klingenberg, 2011). Procrustes

230 coordinates were used in further analysis of shape changes. The data used for statistical analysis

231 are accessible as supplementary material.

232 Statistical analyses

Peer] reviewing PDF | (2020:11:55888:2:1:REVIEW 1 May 2021) 
234 levels of ossification) of analyzed bones. William's correction for small sample size was applied.

235 Multiple correspondence analysis (MCA) was performed to explore the potential influence of

236 thiourea on bone ossification and development.

237 We used PCA to explore and visualize variation in skull shape. To analyze possible 238 differences in skull shape, Procrustes distances between mean shapes of the three analyzed 239 groups were calculated. Permutation tests with 1000 iterations were performed to obtain the 240 pairwise difference between the mean values of cranium shape.

241 The Statistica ${ }^{\circledR} 10.0$ computer package (StatSoft, Inc., Tulsa, OK) was used in

242 performing MCA. The G test was performed using Microsoft Excel. Landmark-based analysis, 243 and graphic visualizations were run in the Morpho J software package (Klingenberg, 2011).

\section{Results}

245 Stage 62

246 At ontogenetic stage 62, all analyzed bones were present in all examined individuals. The 247 premaxilla and vomer were completely ossified in all three groups. The other analyzed bones 248 (exoccipital, frontal, palatopterygoid, parasphenoid, parietal and squamosal) were partially ossified with subtle differences in the level of ossification among individuals from all three analyzed groups with no statistically significant difference among groups ( $G$ test, $p>0.1$ in all

251 comparisons; see Table 1). shape between the tested groups for either the dorsal or the ventral cranium (Table 2). 
At this ontogenetic stage, the frontal, parietal and squamosal bones had the same level of

256

257

ossification in all three groups. The other analyzed bones (nasal, maxilla, prefrontal,

palatopterygoid, pterygoid and vomer) differed in frequencies of the observed level of

ossification states ( $\mathrm{G}$ test, $\mathrm{p}<0.001$; see Table 1). Between-group comparisons showed that the control group differed from both thiourea groups $(\mathrm{G}$ test, $\mathrm{p}<0.001$ for the analyzed bones; see

Table 3), while there were no differences between the high and low thiourea concentration groups ( $\mathrm{G}$ test, $\mathrm{p}>0.2$ for the analyzed bones; see Table 3 ).

At the metamorphic stage, the first axes obtained by MCA explained $65.10 \%$ of the total inertia describing the amount of variation (Fig. 2). On the dorsal side, the maxilla, nasal and prefrontal bones were fully developed in the control group, while larvae subjected to treatment with low and high concentrations of thiourea were mainly characterized by retarded development or even absence of the aforementioned bones. On the ventral side of the skull, control individuals were characterized by a fully developed vomer, unlike individuals from the two thiourea-treated groups, which had vomers with numerous resorption pits, the presence of which is characteristic of the larval skull. Complete disintegration of the palatopterygoid into palatal and pterygoid bones was present in the control group, unlike in the thiourea-treated individuals, where the palatopterygoid showed no signs of disintegration.

Permutation tests showed that there was a statistically significant difference in skull shape between larvae from the control group and the two thiourea-treated groups, but there was no statistically significant difference between the groups receiving low and high concentrations of thiourea (Table 2). For the dorsal cranium, the two thiourea groups separated from the control group along the PC1 axis which described $35.15 \%$ of the total variance. Thiourea-treated individuals retained late larval shape with a narrow cranium, especially in the middle part (the 
278 suture between the frontal and parietal bones; landmarks 4 and 5) and the otico-occipital region

279 (landmarks 6, 7, 8, 9 and 10). The anterior part of the skull (on the level of the premaxilla;

280 landmarks 1,2 and 3) is slightly more slender in thiourea-treated individuals compared to those

281 of the control group (Fig. 3). For the ventral cranium, the PC1 axes described 60.73\% of total

282 variation and differences between the two thiourea groups and the control group. The most

283 pronounced characteristic is that thiourea-treated individuals possess an elongated quadratum

284 (landmarks 4 and 5). In the anterior part of the skull (on the level of the premaxilla; landmarks 1,

2852 and 3) and in the cranium base (landmarks 6, 7 and 8), differences between thiourea-treated

286 individuals and the control group are less pronounced (Fig. 3).

287 Discussion

In general, thyroid hormone (TH) plays an important role in many physiological

processes involved in growth, development and behaviour, and it is essential for normal cranial development. Regulation of TH levels is maintained by a negative feedback loop involving the hypothalamus-pituitary-thyroid (HPT) axis (Kim \& Mohan, 2013; Bassett \& Williams, 2016;

TR-beta), which are members of the nuclear receptor (NR) superfamily and are ligand-inducible transcription factors (Terrien \& Prunet, 2013). In amphibians, the expression of TH-alpha is characteristic of the larval period and is relatively constant from hatching to metamorphosis

296 (Terrien \& Prunet, 2013). TR-alpha receptors are widely distributed through all tissue and are present even before the organism has a functional thyroid gland (Gilbert, 2010). Also, thyroid hormone is among the major factors triggering tissue transformation, metabolism, growth, reproduction and metamorphosis in both anurans and urodeles. Therefore, experimental reduction of thyroid function with anti-thyroid substances better known as endocrine disruptors 
301 (substances like propylthiouracil, methimazole and thiourea) chemically blocks the synthesis of

302 TH and causes a delay in larval development and metamorphosis (Gobdon, Goldsmith \&

303 Charipper, 1943; Degitz et al., 2005; Thambirajah et al., 2019). Lowering of the level of TH in

304 the plasma caused by thiourea results in the failure of most TH-dependent events such as

305 ossification of some cranial bones and skull remodeling, for which a high hormonal

306 concentration is required (Smirnov \& Vassilieva, 2005). On the other hand, experiments with

307 exogenous applied TH to amphibian larvae led to precocious ossification of bones (Terry, 1918;

308 Fox \& Irving, 1950; Dent, Kirby-Smith \& Craig, 1955; Dent \& Kirby-Smith, 1963; Kemp \&

309 Hoyt, 1965; Yeatman, 1967; Hanken \& Hall, 1988; Smirnov \& Vassilieva, 2003a). Results

310 obtained in experiments on skull development during anuran metamorphosis revealed that

311 exogenous applied TH initiates precocious ossification by accelerating growth and expanding the

312 calcified matrix within already differentiated ossification centres (Hanken \& Hall, 1988).

\section{Exposure to thiourea during the midlarval period}

314 In the first experiment, larvae were exposed to two different concentrations of thiourea or

315 were not treated during the midlarval period (from the stage when hind limb digits began to

316 develop to the stage of fully developed limbs, digits and tail). The most notable result is that

317 thiourea does not have such a dramatic effect on skull development during the midlarval period

318 compared to skull development during metamorphosis. All analyzed cranial elements are present

319 without any missing or resorbed ones, with a similar level of ossification in all individuals.

320 Individuals from all analyzed groups had similar skull shape at stage 62, which indicates that

321 larval cranial development proceeds similarly regardless of different TH levels during the

322 midlarval period. This is in keeping with the results of previous studies treating thiourea as an

323 endocrine disruptor that blocks the synthesis of $\mathrm{TH}$. 

independent, but this independence does not mean that early-appearing bones are insensitive to $\mathrm{TH}$, and even an undetectably low concentration of thyroid hormones in the blood was sufficient

327 for regulation of their development. As we mentioned before, thiourea does not completely block activity of the thyroid gland, and a low level of thyroid hormones is probably still present in the blood. In comparison with early larval stages, during the midlarval period TH responsiveness increased and cranial bones became more TH dependent, which means that a higher hormone concentration in the blood plasma is required. For example, under conditions of different hormonal regimes, appearance and differentiation of early-appearing bones (vomer and palatopterygoid) proceed as in natural development during the early and midlarval period, but during metamorphosis their further development and rearrangements transpire differently, depending on the hormonal level (Smirnov \& Vassilieva, 2003a). and rising concentrations of $\mathrm{TH}$ circulating in the blood induce skull remodeling via bone formation, resorption and transformation (Smirnov \& Vassilieva, 2003a). A study on the plethodontid salamander Eurycea bislenata using radioimmunoassay (RIA) revealed low levels of TH in pre-and post-metamorphic stages, with a peak hormone level at the metamorphic stage (Alberch et al.,1986). Similar results were obtained for other urodeles (Eagleson \& McKeown, 1978; Larras-Regard, Taurog \& Dorris, 1981), but not for the axolotl Ambystoma mexicanum, which has an unusual plasma TH profile (a low level of circulating TH, low 5'- deiodinase activity and a low $\mathrm{TH}$ receptor number) and typically does not undergo a metamorphosis. In our experiment, individuals from the control group at the metamorphic stage display the normal skull 
347 development expected for the late larval period and remodeling during metamorphosis. On the

348 other hand, individuals from both thiourea-treated groups (low and high concentrations) retain

349 the late larval skull without remodeling. Based on the results obtained in determination of

350 ossification levels, it can be asserted that members of the control group have fully developed and

351 ossified TH-dependent late-appearing bones (maxilla, nasal and prefrontal), unlike members of

352 the treatment groups, which display serious retardation or even a lack of bone ossification.

353 Complete disintegration of the palatopterygoid was present in the control group, unlike in

354 thiourea-treated individuals where the palatopterygoid failed to disintegrate into the palatal and

355 pterygoid portions. Larval organization of the vomer was retained in thiourea-treated larvae.

356 During ontogeny, the greatest changes of skull shape take place during metamorphosis.

357 Larvae treated with two different thiourea concentrations had similar skull shape, significantly

358 different from skull shape in members of the control group. All goitrogenic larvae retained the

359 same skull shape as at the late larval stage or the stage of the onset of metamorphosis, even

360 though they were at the same age as larvae of the control group. Differences between the control

361 and thiourea-treated groups in the level of ossification, organization of the skull and its shape

362 indicate that these processes are TH-dependent and that their development was halted in

363 thiourea-treated larvae.

364 Our results confirmed that late-appearing bones (maxilla, nasal and prefrontal) are highly

365 TH-dependent in Triturus newts. It is interesting that these three bones have different TH

366 sensitivity in some other Urodela species. For example, all three bones are slightly TH-

367 dependent in Salamandrela keyserlingi, moderately TH-dependent in Ambystoma mexicanum,

368 and highly TH-dependent in Lissotriton vulgaris (Smirnov, Vassilieva \& Merkulova, 2011). The

369 obtained findings indicate the presence of interspecific variation in the TH-dependence of skull 
370 development in various species of Urodela. Rose (1996) proposed that in addition to changes in

371 the level of the TH in the blood and different rate of thyroid gland activity, the evolution of

372 Urodela was accompanied by changes in the TH dependence of some cranial bones. Also,

373 previous studies showed that skull remodeling during metamorphosis is dependent on the applied

374 dosage of $\mathrm{TH}$, which points to interspecific and individual variation of bone $\mathrm{TH}$ sensitivity

375 (Rose, 1999, 2003). However, in our study two applied thiourea concentrations gave essentially

376 the same results in regard to the ossification level and skull remodeling, indicating that the

377 difference in concentrations was insufficient to induce significant differences in the level of bone

378 ossification and/or skull shape between individuals from the two thiourea-treated groups. Also,

379 we examined histological sections of the thyroid glands of larvae that we included in this study

380 (unpublished data). The thyroid gland of larvae treated during the late larval stage with both

381 concentrations of thiourea showed notable hypertrophy and hyperplasia of thyroid follicles,

382 which indicates that the thyroid glands were exhausted and TH synthesis was inhibited.

383 Our study confirms that in Triturus newts a deficiency in TH delays metamorphosis and

384 induces retention of larval skull characteristics (the level of ossification of cranial elements and

385 skull shape), the main feature of paedomorphic species. Paedomorphosis had significant effects

386 on skull bone development in smooth (Lissotriton sp.) and alpine (Ichtyosaura sp.) newts, with

387 inter- and intraspecific variation in ossification levels of cranial elements. However, most adult

388 paedomorphic individuals retain the same cranial skeletal organization as at the late larval stages

389 (Djorović \& Kalezić, 2000; Ivanović et al., 2014). Further studies of metamorphic and post-

390 metamorphic development of newts treated with different concentrations of thiourea during

391 ontogeny could clarify which TH levels are sufficient for metamorphosis, delay metamorphosis

392 or completely block it and lead to paedomorphic adult individuals. 


\section{Conclusion}

Exposure to thiourea during the midlarval period has no very dramatic effect on the level

of ossification of early-appearing cranial bones and does not affect the general skull shape in

Triturus newts. Our results confirmed that early-appearing cranial ossification is TH-

independent, but this independence does not mean that early-appearing bones are insensitive to

TH. At the metamorphic stage, TH-dependent bones play a major role in establishment of skull

shape via the level of cranial ossification, which is highly responsive to thiourea exposure during

endogenous $\mathrm{TH}$, and optimal $\mathrm{TH}$ values are required to maintain the normal rate of skeletal influence of thiourea as an endocrine disruptor at the late larval period and during

metamorphosis between the control and thiourea-treated groups may cause different fitness of

the groups. Future studies could explain how these differences are reflected in post-metamorphic development and what their main consequences are.

407

408

409

410

411

412

\section{Acknowledgements}

We would like to thank many undergraduate students of the Faculty of Biology,

University of Belgrade, for their technical assistance during the experiment.

\section{References}

Arntzen JW, Wielstra B, Wallis GP. 2014. The modality of nine Triturus newt hybrid zones assessed with nuclear, mitochondrial and morphological data. Biological Journal of the Linnean Society 113: 604-622 DOI: 10.1111/bij.12358. 
416 Arntzen JW, Üzüm N, Ajduković MD, Ivanović A, Wielstra B. 2018. Absence of heterosis in 417 hybrid crested newts. PeerJ 6:e5317 DOI: 10.7717/peerj.5317.

418 Alberch P, Gale EA, Larsen PR. 1986. Plasma T4 and T3 levels in naturally metamorphosing

419 Eurycea bislineata (Amphibia; Plethodontidae). General and Comparative

420 Endocrinology 61: 153-163 DOI: 10.1016/0016-6480(86)90261-3.

421

422

423

424

425

426

427

428

429

430

431

432

433

434

435

436

437

438

Altwegg R. 2003. Multistage density dependence in an amphibian. Oecologia. 136: 46-50 DOI: 10.1007/s00442-003-1248-x.

Bassett JD, Williams GR. 2016. Role of thyroid hormones in skeletal development and bone maintenance. Endocrine Reviews 37: 135-187 DOI: 10.1210/er.2015-1106.

Brown DD. 1997. The role of thyroid hormone in zebrafish and axolotl development. Proceedings of the National Academy of Sciences of the United States of America 94: 13011-13016 DOI: 10.1073/pnas.94.24.13011.

Chakraborty A, Sarkar D, Dey P, Chandra KA. 2018. New insights into morphological, stereological and functional studies of the adrenal gland under exposure to the potent goitrogen thiourea. Interdisciplinary Toxicology 11: 38-44 DOI: 10.2478/intox-20180005.

Degitz SJ, Holcombe GW, Flynn KM, Kosian PA, Korte JJ, Tietge JE. 2005. Progress towards development of an amphibian-based thyroid screening assay using Xenopus laevis. Organismal and thyroidal responses to the model compounds 6-propylthiouracil, methimazole and thyroxine. Toxicological Science 87: 353-364 DOI: 10.1093/toxsci/kfi246.

Dent JN, Kirby-Smith JS, Craig DL. 1955. Induction of metamorphosis in Gyrinophilus palleucus. Anatomical Record 121:429. 
439 Dent JN, Kirby-Smith JS. 1963. Metamorphic physiology and morphology of the cave

440

441

442

443

444

445

446

447

448

449

450

451

452

453

454

455

456

457

458

459

460 salamander Gyrinophilus palIeucus. Copeia 1963:119-130 DOI: 10.2307/1441279.

Dingerkus G, Uhler LD. 1977. Enzyme clearing of Alcian blue-stained whole small vertebrates for demonstration of cartilage. Stain Technology 52: 229-232 DOI: 10.3109/10520297709116780.

Djorović A, Kalezić ML. 2000. Paedogenesis in European newts (Triturus: Salamandridae): cranial morphology during ontogeny. Journal of Morphology 243: 127-139 DOI: 10.1002/(SICI)1097-4687(200002)243:2<127: AID-JMOR2>3.0.CO;2-0.

Dodd MHI, Dodd JM. 1976. Biology of metamorphosis. In: Lofts B, ed. Physiology of the Amphibia. New York: Academic, 467-599.

Dryden IL, Mardia KV. 1998. Statistical Shape Analysis. New York: Wiley.

Eagleson GW, McKeown B.A. 1978. Changes in thyroid activity of Ambystoma gracile (Bird) during different larval transforming and postmetamorphic phases. Canadian Journal of Zoology 56: 1377-1381.

Engler H, Taurog A, Nakashima T. 1982. Mechanism of inactivation of thyroid peroxidase by thioureylene drugs. Biochemical Pharmacology 31: 3801 DOI: 10.1016/00062952(82)90296-9.

Fox E, Irving JT. 1950. The effect of thyroid hormone on the ossification of the femur in Xenopus laevis tadpoles. The South African Journal of Medical Sciences 15: 11-14

Gilbert SF. 2010. Developmental biology. Sunderland: Sinauer Associates, Inc.

Glücksohn S. 1931. Äußere Entwicklung der Extremitäten und Stadieneinteilung der Larvenperiode von Triton taeniatus Leyd. und Triton cristatus Laur. Wilhelm Roux' 
461

462

463

464

465

466

467

468

469

470

471

472

473

474

475

476

477

478

479

480

481

Archiv für Entwicklungsmechanik der Organismen 125: 341-405 DOI:

10.1007/bf00576359.

Gobdon AS, Goldsmith ED, Charipper HA. 1943. Effect of thiourea on the development of the amphibian. Nature 152: 504-505 DOI: 10.1038/152504a0.

Hanken J, Hall BK. 1988. Skull development during anuran metamorphosis. Anatomy and Embryology 178: 219-227 DOI: 10.1007/BF00318225.

Hanken J, Summers CH. 1988. Skull development during anuran metamorphosis. III. Role of thyroid hormone in chondrogenesis. Journal of Experimental Zoology 246: 156-170 DOI: $10.1002 /$ jez.1402460208.

Ivanović A, Cvijanović M, Denoël M, Slijepčević M, Kalezić ML. 2014. Facultative paedomorphosis and the pattern of intra-and interspecific variation in cranial skeleton: lessons from European newts (Ichthyosaura alpestris and Lissotriton vulgaris). Zoomorphology 133: 99-109 DOI: 10.1007/s00435-013-0202-7.

Kemp NE, Hoyt JA. 1965. Influence of thyroxine on ossification of the femur in Rana pipiens. Journal of Cell Biology 27: 51A.

Kim HY, Mohan S. 2013. Role and mechanisms of actions of thyroid hormone on the skeletal development. Bone Research 1: 146-161

Klingenberg CP. 2011. MorphoJ: an integrated software package for geometric morphometrics. Molecular Ecology Resources 11: 353-357 DOI: 10.1111/j.1755-0998.2010.02924.x.

Kollros JJ. 1961. Mechanisms of amphibian metamorphosis: hormones. American Zoologist 1: 107-114. 
482 Krishnapriya MV, Arulvasu C, Sheeba P, Sujitha CS, Neethu PG. 2014. Influence of elemental

483

484

485

486

487

488

489

490

491

492

493

494

495

496

497

498

499

500

501

502

503

504 iodine and thiourea on metamorphosis of Philautus sp. Journal of Advanced Botany and Zoology 4: 1-6.

Larras-Regard E, Taurog A, Dorris M. 1981. Plasma T4 and T3 levels in Ambystoma tigrinum at various stages of metamorphosis. General and Comparative Endocrinology 43: 443-450 DOI: $10.1016 / 0016-6480(81) 90228-8$.

Lynn WG. 1948. The effects of thiourea and phenylthiourea upon the development of Eleutherodactylus ricordii. Biological Bulletin 94: 1-15 DOI: 10.2307/1538202.

Norman MF, Carr JA, Norris DO. 1987. Adenohypophysial-thyroid activity of the tiger salamander, Ambystoma tigrinum, as a function of metamorphosis and captivity. Journal of Experimental Zoology 242: 55-66 DOI: 10.1002/jez.1402420108.

Ortiga-Carvalho TM, Chiamolera MI, Pazos-Moura CC, Wondisford FE. 2016.

Hypothalamus-pituitary-thyroid axis. Comprehensive Physiology 6: 1387-1428 DOI: 10.1002/cphy.c150027.

Regard E, Taurog A, Nakashima T. 1978. Plasma thyroxine and triiodothyronine levels in spontaneously metamorphosing Rana catesbeiana tadpoles and in adult anuran amphibia. Endocrinology 102: 674-684 DOI: 10.1210/endo-102-3-674.

Rohlf FJ. 2005. TpsDig 2.04. Available at http://life.bio.sunysb.edu/morph

Rohlf FJ, Slice DE. 1990. Extensions of the Procrustes method for the optimal superimposition of landmarks. Systematic Zoology 39: 40-59 DOI: 10.2307/2992207.

Rose CS. 1995a. Skeletal morphogenesis in the urodele skull: II. Effect of developmental stage in TH-induced remodeling. Journal of Morphology 223: 149-166 DOI: 10.1002/jmor.1052230204.

Peer] reviewing PDF | (2020:11:55888:2:1:REVIEW 1 May 2021) 
505 Rose CS. 1995b. Skeletal morphogenesis in the urodele skull: III. Effect of hormone dosage in 506 TH-induced remodeling. Journal of Morphology 223: 243-261 DOI:

507 10.1002/jmor.1052230303.

508

509

510

511

512

513

514

515

516

517

518

519

520

521

522

523

524

525

526

Rose CS. 1996. An endocrine-based model for developmental and morphogenetic diversification in metamorphic and paedomorphic urodeles. Journal of Zoology 239: 253-284 DOI: 10.1111/j.1469-7998.1996.tb05451.x.

Rose CS. 1999. Hormonal control in larval development and evolution. In: Hall BK, Wake MH, eds. Origin and Evolution of Larval Forms. New York: Academic Press, 167-216.

Rose CS. 2003. The developmental morphology of salamander skull. In: Heatwole H, Davies M, eds. Amphibian Biology. Australia: Surrey Beatty and Sons. Pty. Ltd, 1684-1781.

Rose CS, Reiss JO. 1993. Metamorphosis and the vertebrate skull: ontogenetic patterns and developmental mechanisms. In: Hanken J, Hall BK, eds. The Skull Development. Chicago: University Chicago Press, 289-346.

Sheets HD. 2000. IMP, Integrated Morphometrics Package. Available at http://www3.canisius.edu/ sheets/morphsoft.html

Sheikh EM, McGregor AM. 1997 Antithyroid drugs: their mechanism of action and clinical use. In: Weetman AP, Grossman A, eds. Pharmacotherapeutics of the Thyroid Gland. Handbook of Experimental Pharmacology. Berlin, Heidelberg, 189-206 DOI: 10.1007/978-3-642-60709-7_8.

Smirnov SV, Vassilieva AB. 2003a. Skeletal and dental ontogeny in the smooth newt, Triturus vulgaris (Urodela: Salamandridae): role of thyroid hormone in its regulation. Russian Journal of Herpetology 10: 93-110. 
527 Smirnov SV, Vassilieva BA. 2003b. Bony skull of the smooth newt Triturus vulgaris (Amphibia,

528 Urodela: Salamandridae) and the role of thyroid hormones in its ossification. Doklady

529 Biological Sciences 388: 73-75 DOI: 10.1023/A:1022416530987.

530 Smirnov SV, Vassilieva BA. 2005. Skull development in normal, TH-exposed, and goitrogen-

531 treated axolotls, Ambystoma mexicanum. Russian Journal of Herpetology 12: 113-126.

532 Smirnov SV, Vassilieva, BA, Merkulova MK. 2011. Thyroid hormone mediation in skull

533 development of Siberian newt, Salamandrella keyserlingi (Urodela: Hynobiidae), with

534 comparison to other species. Russian Journal of Herpetology 18: 203-209.

535 Steinfartz S, Vicario S, Arntzen JW, Caccone A. 2007. A Bayesian approach on molecules and

536 behavior: reconsidering phylogenetic and evolutionary patterns of the Salamandridae

537 with emphasis on Triturus newts. Journal of Experimental Zoology 306: 139- 162. DOI:

$538 \quad 10.1002 /$ jez.b.21119.

539 Steinmetz CH. 1950. Some effects of 2-thiouracil on Rana clamitans larvae. Proceedings of the

540 Indiana Academy of Science 60: 324-2.

541 Sternberg RM, Thoemke KR, Korte JJ, Moen SM, Olson JM, Korte L, Tietge JE, Degitz SJ Jr.

542 2011. Control of pituitary thyroid-stimulating hormone synthesis and secretion by thyroid

543 hormones during Xenopus metamorphosis. General and Comparative Endocrinology

$544 \quad$ 173: 428-437 DOI: 10.1016/j.ygcen.2011.06.020.

545 Takamura AE, de Oliveira MA, Vigoya AA, De Stéfani MV, Ribeiro Filho OP. 2020. Thiourea

546 effects on the metamorphosis and development of bullfrog tadpole (Lithobates

547 catesbeianus). International Journal of Research in Engineering and Science 8: 30-41. 
548 Terrien X, Prunet P. 2013. Crossregulation of the thyroid hormone and corticosteroids in

549 amphibians and fish: the effects of endocrine disruption. Insights from Veterinary

$550 \quad$ Medicine 27: 241-254 DOI: 10.5772/54314.

551 Terry GS. 1918. Effects of the extirpation of the thyroid gland upon ossification in Rana pipiens.

$552 \quad$ Journal of Experimental Zoology 24: 567-581 DOI: 10.1002/jez.1400240306.

553 Thambirajah AA, Koide EM, Imbery JJ, Helbing CC. 2019. Contaminant and environmental

554 influences on thyroid hormone action in amphibian metamorphosis. Frontiers in

555 Endocrinology 10: 276 DOI:10.3389/fendo.2019.00276.

556 Vučić T, Vukov TD, Tomašević Kolarov N, Cvijanović M, Ivanović A. 2018. The study of

557 larval tail morphology reveals differentiation between two Triturus species and their

558 hybrids. Amphibia-Reptilia 39: 87-97 DOI: 10.1163/15685381-17000190.

559 Vučić T, Sibinović M, Vukov TD, Tomašević Kolarov N, Cvijanović M, Ivanović A. 2019.

560 Testing the evolutionary constraints of metamorphosis: The ontogeny of head shape in

561 Triturus newts. Evolution 73: 1253-1264 DOI: 10.1111/evo.13743.

562 Wegner S, Browne P, Dix D. 2016. Identifying reference chemicals for thyroid bioactivity

563 screening. Reproductive Toxicology 65: 402-413 DOI: 10.1016/j.reprotox.2016.08.016.

564 Wheeler AJ. 1953. Temporal variations in histological appearance of thyroid and pituitary of

565 Salamanders treated with thyroid inhibitors. Biological Bulletin 104: 250-262 DOI:

566 $10.2307 / 1538798$.

567 White BA, Nicoll CS. 1981. Hormonal control of amphibian metamorphosis. In: Frieden E, 
569 Wielstra B, Arntzen JW. 2011. Unraveling the rapid radiation of crested newts (Triturus cristatus

$570 \quad$ superspecies) using complete mitogenomic sequences. BMC Evolutionary Biology 11: 1-

$571 \quad 8$ DOI: $10.1186 / 1471-2148-11-162$.

572 Wielstra B, Burke T, Butlin RK, Arntzen JW. 2017. A signature of dynamic biogeography:

573 enclaves indicate past species replacement. Proceedings of the Royal Society of London.

$574 \quad$ Series B: Biological Sciences 284:20172014 DOI: 10.1098/rspb.2017.2014.

575 Wielstra B, McCartney-Melstad E, Arntzen JW, Butlin RK, Shaffer HB. 2019. Phylogenomics

576 of the adaptive radiation of Triturus newts supports gradual ecological niche expansion

577 towards an incrementally aquatic lifestyle. Molecular Phylogenetics and Evolution

$578 \quad$ 133:120-127 DOI: 10.1016/j.ympev.2018.12.032.

579 Yeatman HC. 1967 Artificially metamorphosed neotenic cave salamanders.

$580 \quad$ Journal of the Tennessee Academy of Science 42: I6-22.

581 


\section{Figure legends}

583 Fig.1. Configuration of two-dimensional landmarks for the dorsal and ventral skull at 584 metamorphic stages.

585 Fig.2. Graphic presentation of the positions of control and two thiourea treatment groups (red 586 squares) relative to the first two axes obtained by MCA on skull character state scores (blue 587 circles) for the metamorphic stage. The inertia represents the amount of variation of skull 588 character state scores described by each axis.

589 Fig.3. Position of control and two thiourea treatment groups (A and B) with deformation grids as 590 an illustration of shape changes (C-F) for the dorsal and ventral cranium in morphospace defined 591 by the first two PC axes at the metamorphic stage. In deformation grids (C-F), blue circles 592 represent each landmark and the set of landmarks describe mean cranial shape. Straight blue 593 lines describe the shift in positions of landmarks from the mean shape to the target shape (C and $594 \mathrm{E}$ - positive part of the axis, $\mathrm{D}$ and $\mathrm{F}$ - negative part of the axis).

595

596

597

598

599

600

601

602

603

604 
605 Tables

606 Table 1: Differences between groups in the frequencies of ossification level states at ontogenetic 607 stage 62 and the metamorphic stage.

608 Table 2: Procrustes distances (PD) and $p$-values from permutation tests (with 1000 permutation 609 rounds).

610 Table 3: Between-group comparisons of the frequencies of ossification level states at the 611 metamorphic stage. The vomer, palatopterygoid and pterygoid bones have the same level of 612 ossification in high and low thiourea-treated groups. 
Figure 1

Configuration of two-dimensional landmarks for the dorsal and ventral skull at metamorphic stages 


\section{metamorphic stage}

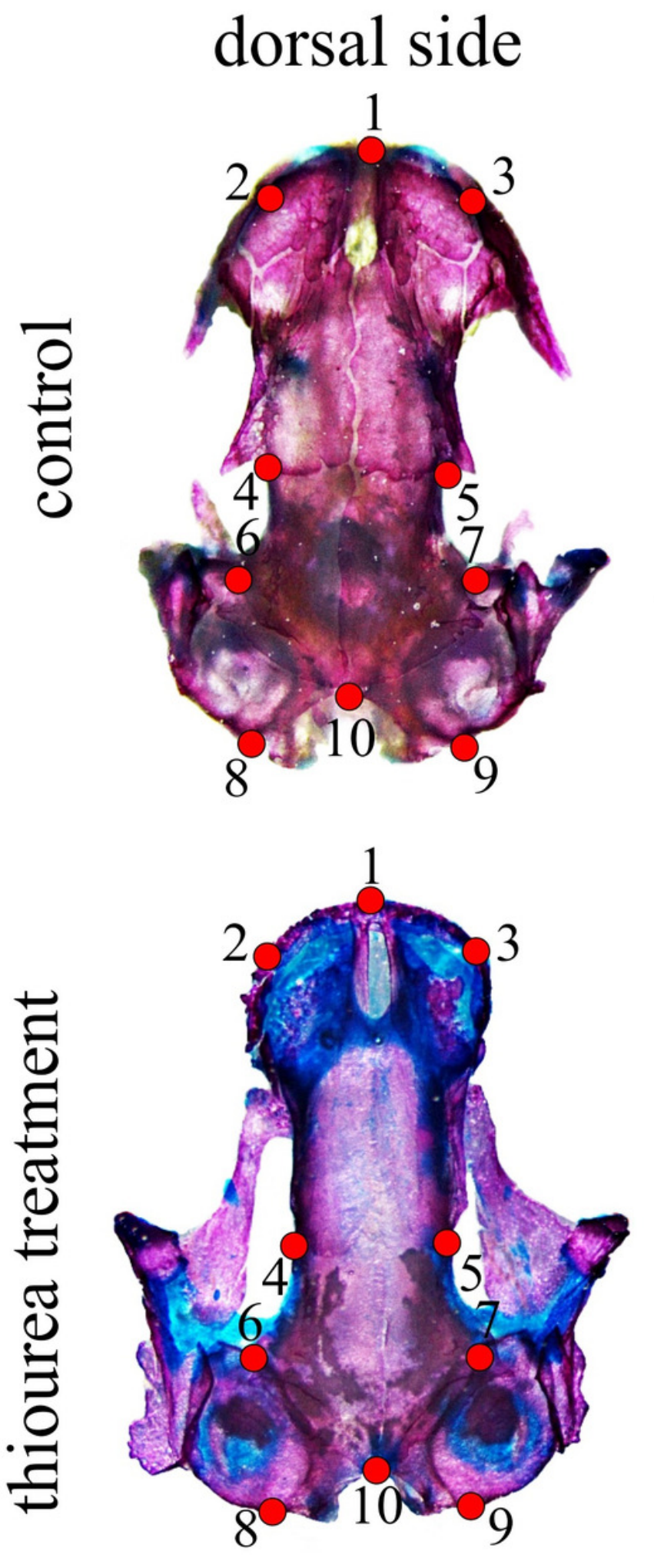

ventral side
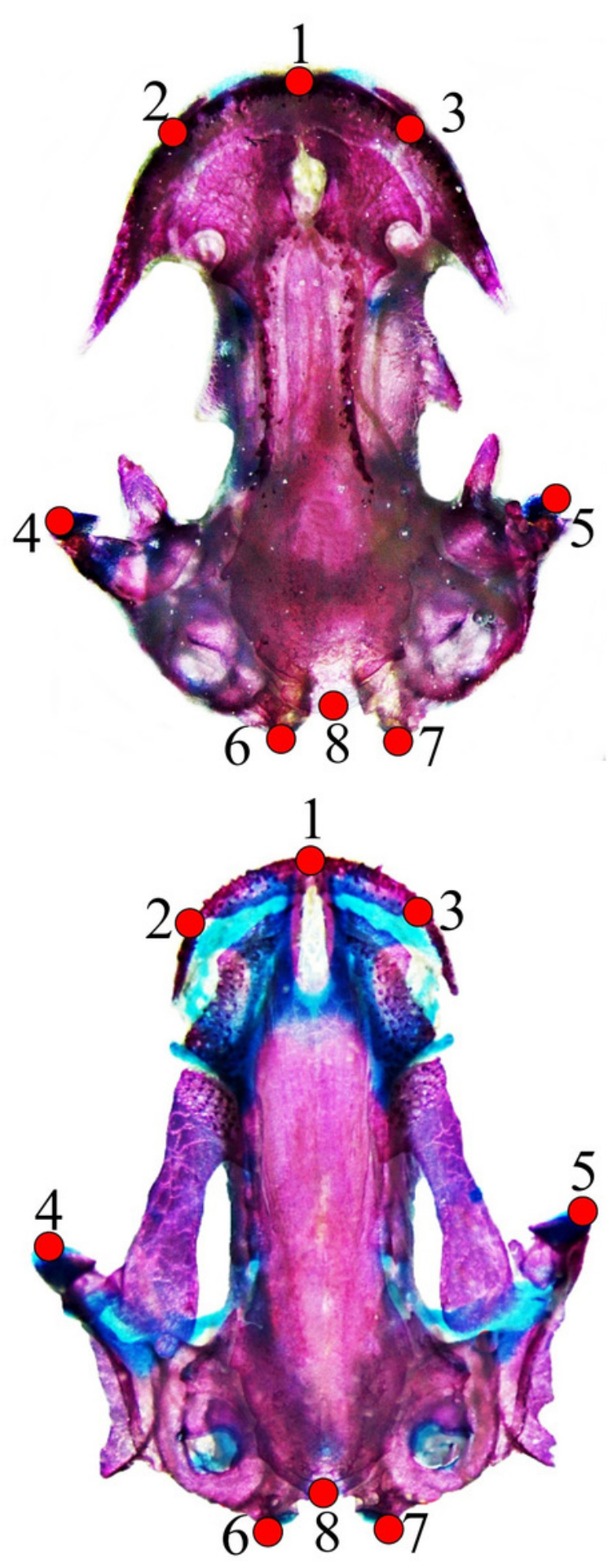
Figure 2

Multiple Correspondence Analysis of skull ossification

Graphic presentation of the positions of control and two thiourea treatment groups (red squares) relative to the first two axes obtained by MCA on skull character state scores (blue circles) for the metamorphic stage. The inertia represents the amount of variation of skull character state scores described by each axis.

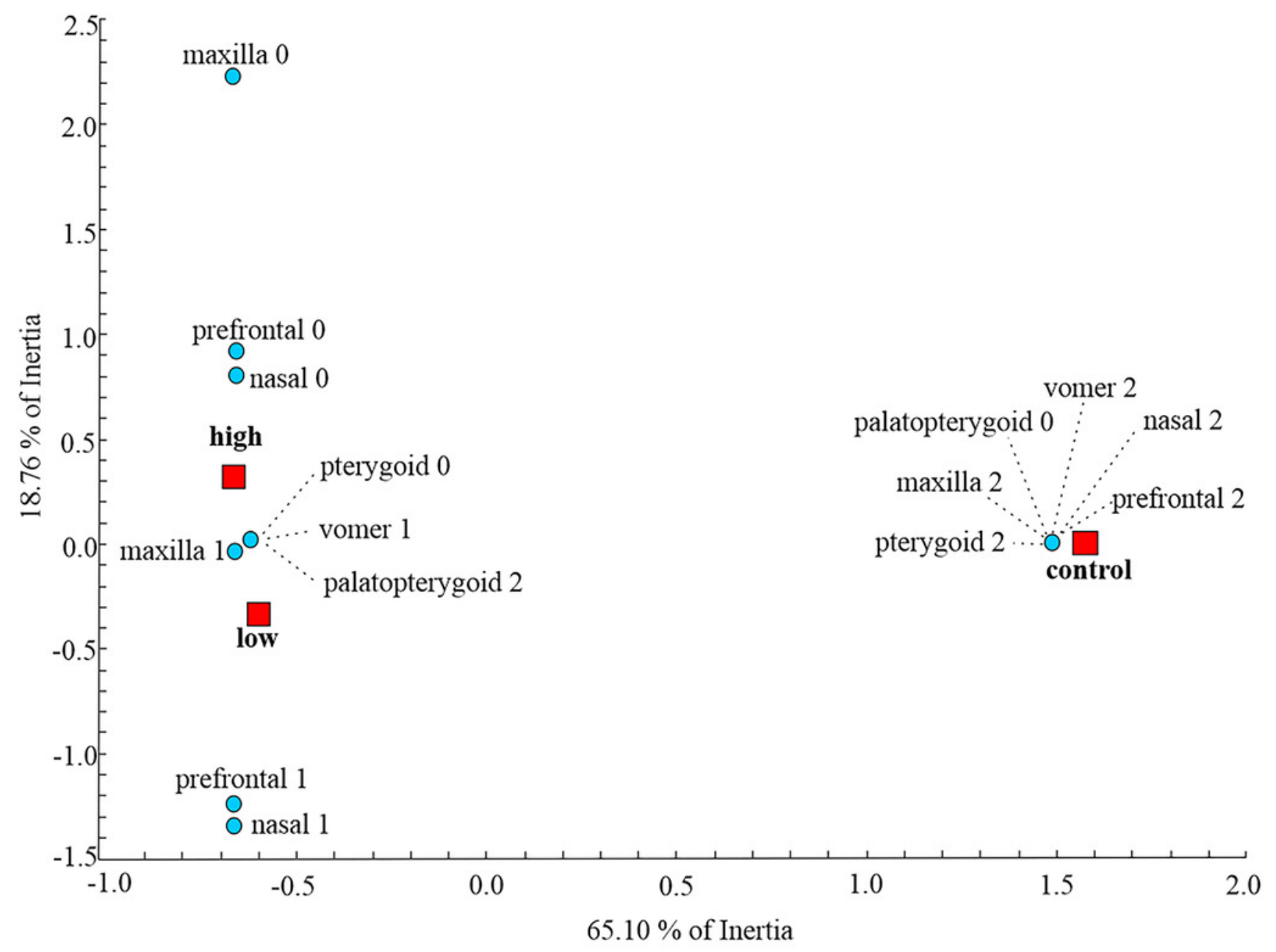


Figure 3

Principal Component Analysis and shape changes of the skull

Position of control and two thiourea treatment groups ( $A$ and $B$ ) with deformation grids as an illustration of shape changes (C-F) for the dorsal and ventral cranium in morphospace defined by the first two PC axes at the metamorphic stage. In deformation grids (C-F), blue circles represent each landmark and the set of landmarks describe mean cranial shape. Straight blue lines describe the shift in positions of landmarks from the mean shape to the target shape ( $C$ and $E$ - positive part of the axis, $D$ and $F$ - negative part of the axis).
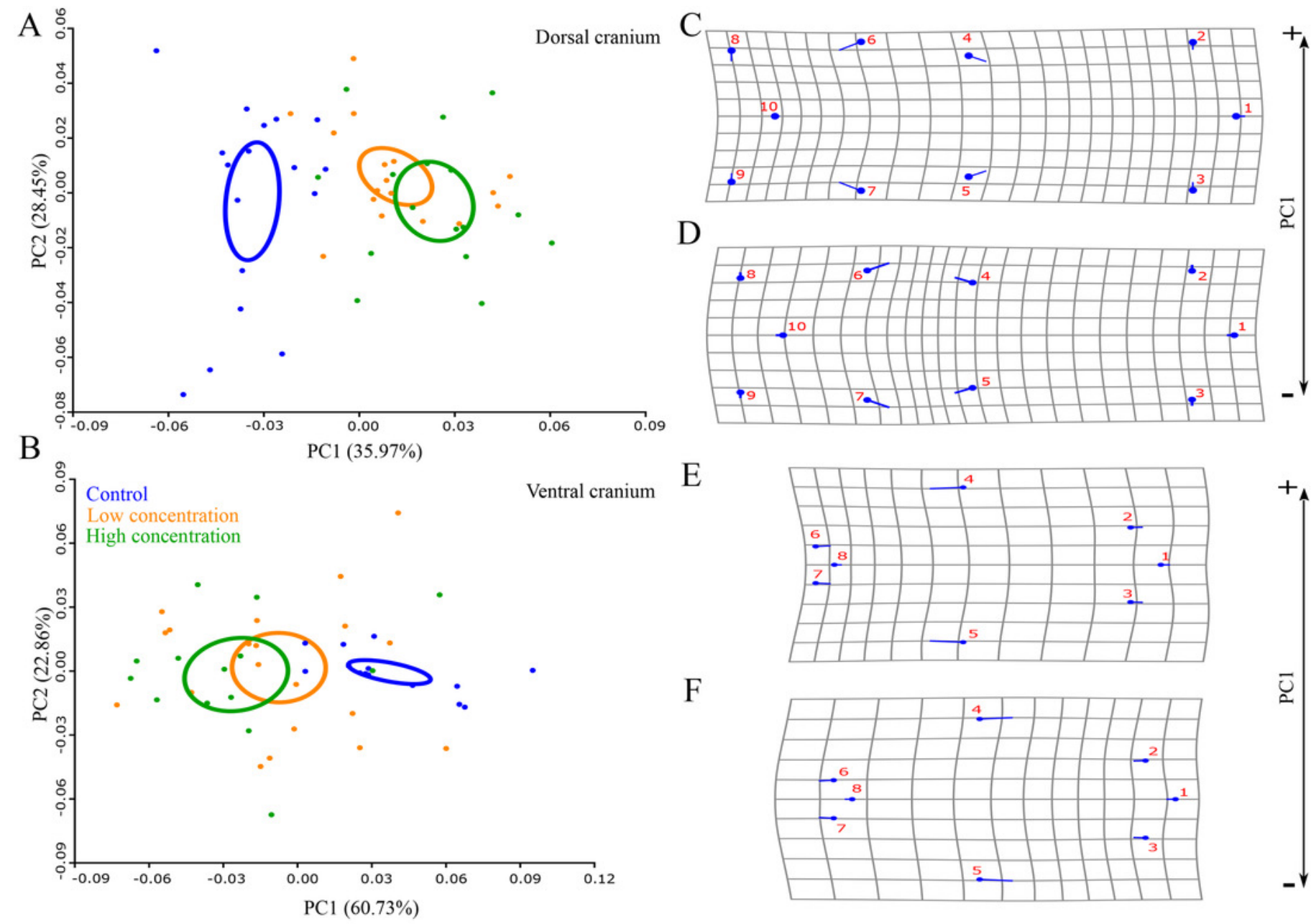

$\mathrm{E}$

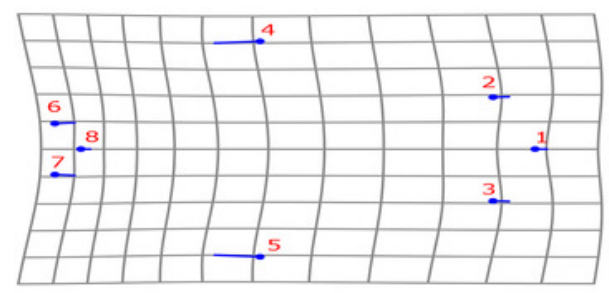

$\mathrm{F}$

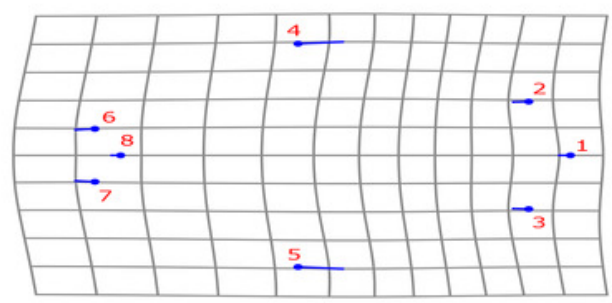

$\underset{\swarrow}{\bar{\swarrow}}$ 


\section{Table 1 (on next page)}

Differences between groups in the frequencies of ossification level states at ontogenetic stage 62 and the metamorphic stage 
1 Table 1. Differences between groups in the frequencies of ossification level states at ontogenetic 2 stage 62 and the metamorphic stage.

\begin{tabular}{lcclcc}
\hline Stage 62 & G test & $\mathrm{p}$ & Metamorphic & G test & $\mathrm{p}$ \\
\hline frontal & 0.35 & 0.841 & nasal & 51.81 & $<0.001$ \\
parietal & 0.35 & 0.841 & prefrontal & 50.87 & $<0.001$ \\
squamosal & 0.91 & 0.631 & maxilla & 39.61 & $<0.001$ \\
exoccipital & 3.02 & 0.221 & vomer & 55.63 & $<0.001$ \\
palatopterygoid & 3.36 & 0.187 & palatopterygoid & 55.63 & $<0.001$ \\
parasphenoid & 4.27 & 0.118 & pterygoid & 55.63 & $<0.001$ \\
\hline
\end{tabular}


Table 2 (on next page)

Procrustes distances (PD) and $p$-values from permutation tests (with 1000 permutation rounds). 
1 Table 2. Procrustes distances (PD) and $p$-values from permutation tests (with 1000 permutation 2 rounds).

\begin{tabular}{|c|c|c|c|c|c|c|c|c|c|}
\hline \multirow{3}{*}{\multicolumn{2}{|c|}{ Compared groups }} & \multicolumn{4}{|c|}{ Stage 62} & \multicolumn{4}{|c|}{ Metamorphosis } \\
\hline & & \multicolumn{2}{|c|}{ Dorsal } & \multicolumn{2}{|c|}{ Ventral } & \multicolumn{2}{|c|}{ Dorsal } & \multicolumn{2}{|c|}{ Ventral } \\
\hline & & PD & $\mathrm{p}$ & PD & $\mathrm{p}$ & PD & $\mathrm{p}$ & PD & $\mathrm{p}$ \\
\hline Control & Low & 0.0188 & 0.242 & 0.0129 & 0.685 & 0.0473 & $<0.001$ & 0.0463 & $<0.001$ \\
\hline Control & - High & 0.0265 & 0.131 & 0.0239 & 0.205 & 0.0584 & $<0.001$ & 0.0635 & $<0.001$ \\
\hline Low & High & 0.016 & 0.263 & 0.0223 & 0.111 & 0.0176 & 0.206 & 0.0178 & 0.354 \\
\hline
\end{tabular}

3

4 


\section{Table 3(on next page)}

Between-group comparisons of the frequencies of ossification level states at the metamorphic stage. The vomer, palatopterygoid and pterygoid bones have the same level of ossification in high and low thiourea-treated groups. 
1 Table 3. Between-group comparisons of the frequencies of ossification level states at the

2 metamorphic stage. The vomer, palatopterygoid and pterygoid bones have the same level of 3 ossification in high and low thiourea-treated groups.

4

5

\begin{tabular}{lcccccc}
\hline bones & \multicolumn{2}{c}{ control - high } & \multicolumn{2}{c}{ control - low } & \multicolumn{2}{c}{ high - low } \\
& $\mathrm{G}$ test & $\mathrm{p}$ & $\mathrm{G}$ test & $\mathrm{p}$ & $\mathrm{G}$ test & $\mathrm{p}$ \\
\hline nasal & 37.91 & $<0.001$ & 35.29 & $<0.001$ & 2.77 & 0.251 \\
prefrontal & 38.29 & $<0.001$ & 35.29 & $<0.001$ & 1.95 & 0.378 \\
maxilla & 32.44 & $<0.001$ & 35.94 & $<0.001$ & 1.85 & 0.396 \\
vomer & 39.46 & $<0.001$ & 39.46 & $<0.001$ & - & - \\
palatopterygoid & 39.46 & $<0.001$ & 42.91 & $<0.001$ & - & - \\
pterygoid & 39.46 & $<0.001$ & 42.91 & $<0.001$ & - & - \\
\hline
\end{tabular}

